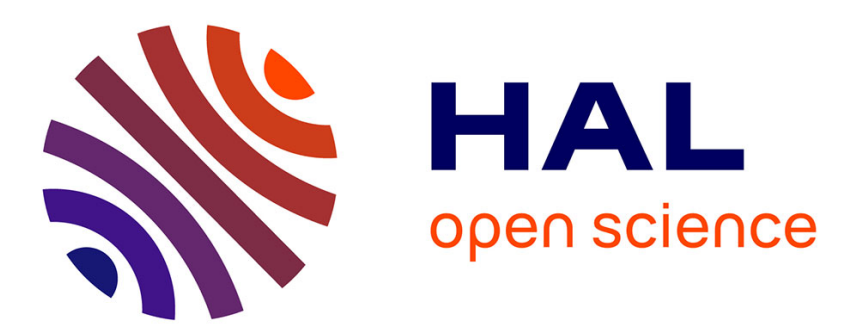

\title{
Wireless Service Providers Pricing Game in Presence of Possible Sponsored Data
}

\author{
Patrick Maillé, Bruno Tuffin
}

\section{To cite this version:}

Patrick Maillé, Bruno Tuffin. Wireless Service Providers Pricing Game in Presence of Possible Sponsored Data. 15th International Conference on Network and Service Management (CNSM), Oct 2019, Halifax, Canada. 10.23919/CNSM46954.2019.9012709 . hal-02004692

\section{HAL Id: hal-02004692 \\ https://hal.inria.fr/hal-02004692}

Submitted on 2 Feb 2019

HAL is a multi-disciplinary open access archive for the deposit and dissemination of scientific research documents, whether they are published or not. The documents may come from teaching and research institutions in France or abroad, or from public or private research centers.
L'archive ouverte pluridisciplinaire HAL, est destinée au dépôt et à la diffusion de documents scientifiques de niveau recherche, publiés ou non, émanant des établissements d'enseignement et de recherche français ou étrangers, des laboratoires publics ou privés. 


\title{
Wireless Service Providers Pricing Game in Presence of Possible Sponsored Data
}

\author{
Patrick Maillé \\ IMT Atlantique \\ 2, rue de la ChÃćtaigneraie, 35576 Cesson Sévigné Cedex, France, \\ patrick.maille@imt.fr \\ Bruno Tuffin \\ Inria, Univ Rennes, CNRS, IRISA \\ Campus Universitaire de Beaulieu, 35042 Rennes Cedex, France \\ bruno.tuffin@inria.fr
}

February 2, 2019

\begin{abstract}
Sponsored data, where content providers have the possibility to pay wireless providers for the data consumed by customers and therefore to exclude it from the data cap, is getting widespread in many countries, but is forbidden in others for concerns of infringing the network neutrality principles. We present in this paper a game-theoretic model analyzing the consequences of sponsored data in presence of competing wireless providers, where sponsoring decided by the content provider can be different at each provider. We also discuss the impact on the proportion of advertising on the displayed content. We show that, surprisingly, the possibility of sponsored data may actually reduce the benefits of content providers and on the other hand increase the revenue of ISPs in competition, with a very limited impact on user welfare.
\end{abstract}

Keywords: Competition, sponsored data, network neutrality, game theory.

\section{Introduction}

Wireless communications are becoming ubiquitous and data are increasingly been consumed through mobile phone; it is for example admitted that mobile data consumption will be seven-fold larger by 2021 than by $2017^{1}$. Though, data plans proposed by Internet Service Providers (ISPs), that is, the wireless

\footnotetext{
${ }^{1}$ see http://www. businessinsider.fr/us/mobile-data-will-skyrocket-700-by-2021-2017-2/ among others
} 
operators, are made of offers with data caps [5], potentially limiting end users consumption. For this reason, in order to be more attractive and gain more in terms of advertising, content providers (CPs) are increasingly thinking of sponsoring data, that is, of paying for the volume of data downloaded by their consumers, therefore excluding their traffic from the data cap. This type of service is active with for example Netflix or Binge-On with T-Mobile, DIRECTV and U-verse Data Free TV with AT\&T. ISPs are even offering capabilities to facilitate access to sponsored data programs by CPs, such as AT\&T with its AT\&T Sponsored Data program ${ }^{2}$, Verizon with FreeBee Data, or Orange with DataMI in France; even third parties are proposing this type of service, such as Aquto.

While a priori interesting for end users, the principle of sponsoring data has been questioned by user associations and some regulators, and is under investigation as a possible infringement of network neutrality rules [4]. Indeed, neutrality rules imposed in many countries state that all "traffic should be treated equally, without discrimination, restriction or interference, independent of the sender, receiver, type, content, device, service or application" (definition from the European parliament on April 3rd, 2014). Offering a differentiated economic treatment to the CPs with respect to others can be considered as a violation of this rule and prevent newcomers not able to afford similar offers from entering the market. Laws to ban it have been imposed in countries such as Canada, Sweden, Hungary, India, Brazil, among others. Europe is putting it in a "grey zone" and let the decision to be taken by national regulatory bodies. A weaker version is when consumers are not differentiated by CPs depending on their origin, here their ISP, hence applying the same sponsoring at all ISPs.

Sponsoring data has recently been subject to modeling and analysis in the literature. We can cite [2] where the model is made of a discrete set of users, a single ISP, and several (complement) CPs; it is then shown that sponsoring can benefit more to users than to CPs. Our model is different in many aspects and incorporates a major component, the negative externality of advertisements on users. It also includes a model of competition between ISPs, the main contribution, with potentially differentiated users, some of them being sponsored while others are not. This competition particularity is also not included in the other works [9], dealing with several substitutable CPs in competition, [11, 12] on including network externalities, or [8] combining sponsored data and caching strategies. The main other work we know involving competition between ISPs is [10] with interesting theoretical results similar to ours, that ISPs may be the ones benefiting the most from sponsoring, but the model does not involve advertising, and includes a to-be-motivated Hotelling model for users' ISP choice. Another reference is [3], using two-sided pricing with benefits to user welfare depending on the proportion of content being sponsored, where the equilibrium prices in the case of many competing ISPs is said to be reduced to a monopoly network.

The present work can be considered as a variation and extension of our

\footnotetext{
${ }^{2}$ See https ://developer.att.com/sponsored-data
} 
previous paper on the topic [7]; the model is very close, but has the following key differences: it first limits the heterogeneity of users to two classes instead of a continuum; this allows to add more tractability to the model and to introduce the pricing game between ISPs that could not be considered in [7]. This last part is the key element here, allowing to compare the output in a competitive environment on revenues and user welfare of the different sponsoring strategies. We also limit the sponsoring level to no sponsoring at all or full sponsoring instead of any proportion, something more representative of what is experienced. Third we add a management cost to ISPs to represent the cost for handling a given amount of data and avoiding congestion. Finally, we keep and highlight the advertising level as a variable of decision for the $\mathrm{CP}$, since it is something likely to change with sponsoring in order to compensate the potential sponsoring costs.

The remaining of the paper is organized as follows. Section 2 recalls the model from [7] with the differences presented in the previous paragraph; we also explain the different layers of decision, with the additional pricing game between ISPs introduced here. Section 3 illustrates the impact of CP decisions on sponsoring and advertising levels on the different actors, for fixed prices. Then Section 4 analyses the upper-level pricing game between ISPs, anticipating the subsequent decision of the CP considered in the previous section; we discuss the obtained numerical results and highlight some counter-intuitive results that may occur, emphasizing the need of scrutiny by regulators. Section 5 then concludes and presents directions for future research.

\section{Model}

The model we summarize here is mainly taken from [7] with restrictions on user heterogeneity and sponsoring possibilities, but includes a management cost for ISPs. This allows more tractability for the analysis of the pricing game between ISPs in Section 4.

The model is made of three different types of actors: users, CPs and ISPs. For generality purposes, the described model is considering $M$ CPs (indexed by $j$ ) and $N$ ISPs (indexed by $i$ ), but we will later limit ourselves to one CP (and two ISPs) without loss of generality under the assumption that CPs are complements.

\section{$2.1 \quad$ Users}

Users are of two classes, characterized by their willingness-to-pay: one class indexed by $h$ and the other by $\ell$, with respective willingness-to-pay for connectivity $\theta_{h} \in \mathbb{R}^{+}$and $\theta_{\ell} \in \mathbb{R}^{+}$, with $\theta_{h}>\theta_{\ell}$. Let $\psi_{h}$ and $\psi_{\ell}$ be the proportion of each class, with $\psi_{h}+\psi_{\ell}=1$.

Users in Class $k(k \in\{h, \ell\})$ need to choose their ISP, but also the volume of CP $j$ data (without advertisements) they will consume if using ISP $i$, which we will denote by $v_{i, j}\left(\theta_{k}\right)$. 
With willingness-to-pay for connectivity $\theta_{k}$ and $p_{i}$ the subscription price at ISP $i$, the "utility for connectivity" would be $\theta-p_{i}$, that we weigh by a factor $a_{i} \geq 0$ representing the reputation of ISP $i$. So the final "utility for connectivity" is $a_{i}\left(\theta-p_{i}\right)$.

Users also gain from using each $\mathrm{CP} j$. Denote by $c_{i, j}$ the cost per unit of volume paid by a user in his data plan. For a user with willingness-topay $\theta \in\left\{\theta_{h}, \theta_{\ell}\right\}$, the marginal valuation is assumed to be linear, $r_{\theta, j}^{\prime}(x)=$ $\left[\theta-\left(\alpha_{j} s_{j}^{2}\right) x\right]^{+}$for the $x$-th unit of useful volume, i.e., without advertising, where $[y]^{+}:=\max (y, 0), \alpha_{j}$ is a fixed parameter, and $s_{j} \geq 1$ corresponds to the relative increase of volume due to advertisement displayed by CP $j$, expressed as the total downloaded volume divided by the volume of "useful" data, excluding ads. Note that the larger $s_{j}$ the larger the dissatisfaction of users, with a square value of $s_{j}$ to later make sure that above one level the loss due to users unpleased by advertisements exceeds the gain from those ads and the inclusion of $\theta$ positively correlates valuation for data and willingness-to-pay for connectivity. This leads to the willingness-to-pay $r_{\theta, j}(x)$ of $[1,6]$ for consuming a volume $x$ of $\mathrm{CP} j$ data over a month:

$$
r_{\theta, j}(x)= \begin{cases}\theta x-\alpha_{j} s_{j}^{2} \frac{x^{2}}{2} & \text { if } x \leq \frac{\theta}{\alpha_{j} s_{j}^{2}} \\ \frac{\theta^{2}}{2 \alpha_{j} s_{j}^{2}} & \text { otherwise. }\end{cases}
$$

The resulting utility for user $\theta$ at ISP $i$ is

$$
U_{i}(\theta):=a_{i}\left(\theta-p_{i}\right)+\sum_{j=1}^{M}\left(r_{\theta, j}\left(v_{i, j}(\theta)\right)-c_{i, j} v_{i, j}(\theta) s_{j}\right),
$$

using the fact that the total downloaded volume is actually $v_{i, j}(\theta) s_{j}$ from the definition of $s_{j}$. Users indeed also download advertisements, which are not differentiated from "real" content by ISPs. CPs are assumed to be independent in terms of content from the additive expression.

It is easy to compute that if User $\theta$ subscribes to ISP $i$, the volume $v_{i, j}(\theta)$ maximizing its utility is the one maximizing $r_{\theta, j}\left(v_{i, j}(\theta)\right)-c_{i, j} s_{j} v_{i, j}(\theta)$, and equals (see [1] if details are needed):

$$
v_{i, j}(\theta)=\left[\frac{\theta-c_{i, j} s_{j}}{\alpha_{j} s_{j}^{2}}\right]^{+}
$$

giving finally

$$
r_{\theta, j}\left(v_{i, j}(\theta)\right)-c_{i, j} s_{j} v_{i, j}(\theta)=\frac{\left(\theta-c_{i, j} s_{j}\right)^{2}}{2\left(\alpha_{j} s_{j}^{2}\right)} 1_{\left\{\theta>c_{i, j} s_{j}\right\}} .
$$

A user with willingness-to-pay $\theta$ will actually choose the ISP $i(\theta)$ yielding the maximal utility when comparing utilities with optimal data volumes. In other words,

$$
i(\theta)=\operatorname{argmax}_{i} U_{i}(\theta)=\operatorname{argmax}_{i} a_{i}\left(\theta-p_{i}\right)+\sum_{j=1}^{M} \frac{\left(\theta-c_{i, j} s_{j}\right)^{2}}{2\left(\alpha_{j} s_{j}^{2}\right)} 1_{\left\{\theta>c_{i, j} s_{j}\right\}}
$$


if the max is non-negative, otherwise $i(\theta)=0$, meaning no subscription at all.

Since we have two classes, that is, two values of $\theta$, determining the ISP to subscribe to, or none, is simple, by comparing the utilities for the $N$ ISPs.

\subsection{CPs}

$\mathrm{CP} j$ is gaining money from advertisement. This gain is assumed linear in terms of the volume of displayed advertisement, with CP-dependent linear parameter $\beta_{j}$. The advertisement volume is the total volume $v_{i(\theta), j} s_{j}$ minus the "real" data volume $v_{i(\theta), j}$, hence $\left(s_{j}-1\right) v_{i(\theta), j}(\theta)$ giving a gain

$$
\begin{aligned}
& \psi_{h} \beta_{j}\left(s_{j}-1\right) v_{i\left(\theta_{h}\right), j}\left(\theta_{h}\right)+\psi_{\ell} \beta_{j}\left(s_{j}-1\right) v_{i\left(\theta_{\ell}\right), j}\left(\theta_{\ell}\right)= \\
& \psi_{h} \beta_{j}\left(s_{j}-1\right)\left[\frac{\theta_{h}-c_{i\left(\theta_{h}\right), j} s_{j}}{\alpha_{j} s_{j}^{2}}\right]^{+}+\psi_{\ell} \beta_{j}\left(s_{j}-1\right)\left[\frac{\theta_{\ell}-c_{i\left(\theta_{\ell}\right), j} s_{j}}{\alpha_{j} s_{j}^{2}}\right]^{+} .
\end{aligned}
$$

$\mathrm{CP} j$ has several decision variables: the advertisement volume increase level $s_{j} \geq 1$, and whether it sponsors or not data usage for each ISP $i$ subscriber. Define $\gamma_{i, j}=1$ if full sponsoring is decided, and $\gamma_{i, j}=0$ if the CP decides not to sponsor. Recall that sponsoring could be an incentive to consume more $\mathrm{CP} j$ content and therefore generate more revenue from advertisement. We want to compare three different sponsoring policies:

1. No sponsored data: $\gamma_{i, j}=0 \forall i, j$;

2. The same data sponsoring level for all ISPs: $\gamma_{i, j}=\gamma_{j} \in\{0,1\} \forall i, j$;

3. A possible differentiation between ISPs, with $\gamma_{i, j} \neq \gamma_{i^{\prime}, j}$ for $i \neq i^{\prime}$.

Denote by $q_{i}$ the unit price ISP $i$ charges users for data (when there is no sponsoring). After data sponsoring, the unit cost experienced by users when consuming data of $\mathrm{CP} j$ is then

$$
c_{i, j}=q_{i}\left(1-\gamma_{i, j}\right) .
$$

So CP $j$ has to pay to each ISP for the proportion of volume it has chosen to sponsor, leading to a price to pay

$$
\begin{aligned}
& \psi_{h} \gamma_{i\left(\theta_{h}\right), j} q_{i\left(\theta_{h}\right)} v_{i\left(\theta_{h}\right), j} s_{j}+\psi_{\ell} \gamma_{i\left(\theta_{\ell}\right), j} q_{i\left(\theta_{\ell}\right)} v_{i\left(\theta_{\ell}\right), j} s_{j} \\
& =\psi_{h} \gamma_{i\left(\theta_{h}\right), j} q_{i\left(\theta_{h}\right)}\left[\frac{\theta_{h}-c_{i\left(\theta_{h}\right), j} s_{j}}{\alpha_{j} s_{j}^{2}}\right]^{+} s_{j}+\psi_{\ell} \gamma_{i\left(\theta_{\ell}\right), j} q_{i\left(\theta_{\ell}\right)}\left[\frac{\theta_{\ell}-c_{i\left(\theta_{\ell}\right), j} s_{j}}{\alpha_{j} s_{j}^{2}}\right]^{+} s_{j} .
\end{aligned}
$$

Compiling all the elements, the revenue of $\mathrm{CP} j$ is

$G_{j}=\psi_{h}\left(\beta_{j}\left(s_{j}-1\right)-\gamma_{i(\theta), j} q_{i\left(\theta_{h}\right)} s_{j}\right) v_{i\left(\theta_{h}\right), j}\left(\theta_{h}\right)+\psi_{\ell}\left(\beta_{j}\left(s_{j}-1\right)-\gamma_{i(\theta), j} q_{i\left(\theta_{\ell}\right)} s_{j}\right) v_{i\left(\theta_{\ell}\right), j}\left(\theta_{\ell}\right)$. 


\section{$2.3 \quad$ ISPs}

ISPs seek to maximize their revenue too. The gain of ISP $i$ comes from subscriptions and consumed data:

$$
\psi_{h}\left(p_{i}+\sum_{j} q_{i} s_{j} v_{i, j}\left(\theta_{h}\right)\right) 1_{\left\{i\left(\theta_{h}\right)=i\right\}}+\psi_{\ell}\left(p_{i}+\sum_{j} q_{i} s_{j} v_{i, j}\left(\theta_{\ell}\right)\right) 1_{\left\{i\left(\theta_{\ell}\right)=i\right\}} .
$$

We here add (or more exactly subtract) a management cost to ensure a given quality $q$ for a demand (volume) $D(q)$ :

$$
f\left(\psi_{h} s_{j} v_{i, j}\left(\theta_{h}\right) 1_{\left\{i\left(\theta_{h}\right)=i\right\}}+\psi_{\ell} s_{j} v_{i, j}\left(\theta_{\ell}\right) 1_{\left\{i\left(\theta_{\ell}\right)=i\right\}}\right)
$$

with the total volume treated by ISP $i$ as the argument of $f$, and $f(x)=\kappa x^{2}$ (that is, convex) to incorporate the fact that the more volume, the more difficult it is to handle traffic/congestion. It gives a revenue

$$
\begin{aligned}
R_{i}= & \psi_{h}\left(p_{i}+\sum_{j} q_{i} s_{j} v_{i, j}\left(\theta_{h}\right)\right) 1_{\left\{i\left(\theta_{h}\right)=i\right\}}+\psi_{\ell}\left(p_{i}+\sum_{j} q_{i} s_{j} v_{i, j}\left(\theta_{\ell}\right)\right) 1_{\left\{i\left(\theta_{\ell}\right)=i\right\}} \\
& -\kappa\left(\psi_{h} s_{j} v_{i, j}\left(\theta_{h}\right) 1_{\left\{i\left(\theta_{h}\right)=i\right\}}+\psi_{\ell} s_{j} v_{i, j}\left(\theta_{\ell}\right) 1_{\left\{i\left(\theta_{\ell}\right)=i\right\}}\right)^{2} .
\end{aligned}
$$

\subsection{Hierarchy of decisions}

Recall that our goal is to compare the impact of the three scenarios of sponsored data when there is a game between ISPs, in order to illustrate its benefits or limits and to provide guidelines to regulators.

Decisions are taken at different time scales

1. ISPs play a (non-cooperative) game, ISP $i$ deciding $q_{i}$; indeed, we assume here that subscription prices are fixed, determined at another level or by regulation;

2. The CP decides its level of sponsoring and of advertising;

3. Users decide which ISP they choose (done by computing the utilities with all options).

The game is played by backward induction: players making decisions at long time scales are assumed to anticipate the decisions made at shorter time scales.

Similarly to [7], we can point out that since CPs are complements, they can be treated independently so considering just one is without loss of generality. We therefore remove the CP-relative indices in the notations defined before. We will also limit ourselves to 2 ISPs, labeled 1 and 2 . 


\section{CP decisions}

This section discusses the CP strategic decisions, that is, the choice of sponsoring or not and the advertisement level, for fixed ISP prices since those prices are determined first. In our numerical experiments, we take $\kappa=1$ (management cost coefficient). Other parameters are $\beta=1, r_{1}=2, r_{2}=1$ (so that ISP 1 has a better reputation and can be considered as an incumbent provider), $p_{1}=0.16$, $p_{2}=0.1, q_{1}=0.2, q_{2}=0.1$ (ISP 1 can charge more, being more attractive in terms of reputation) and $\alpha=1$.

Figures 1 to 4 present the outputs in terms of $s$ when the $\gamma \mathrm{s}$ are optimized.



Figure 1: Optimal $\gamma$ when $\kappa=1.00, \beta=1.00, r_{1}=2.00, r_{2}=1.00, p_{1}=$ $0.16, p_{2}=0.10, q_{1}=0.20, q_{2}=0.10, \alpha=1.00$

Figure 1 shows the evolution of the optimal sponsoring decision in terms of the advertising level. One can check the (still) expected results that the more you advertise (and therefore earn money), the more you are able to sponsor. Moreover, equal sponsoring leads to a decision to sponsor in between the decision for the two class when differentiation is possible. Note that when optimal sponsoring decisions are the same (no sponsoring at all before $s=1.225$ ), all the results outputs shown in next figures are also the same, and after $s=1.425$ the cases of (full) sponsoring also yield the same outputs.

Figure 2 shows the resulting $\mathrm{CP}$ revenue (at optimal $\gamma \mathrm{s}$ ). The more freedom you let on sponsoring, the better it is for the CP (for fixed ISP prices in this section). We can also see for those parameters that for each sponsoring strategy, 


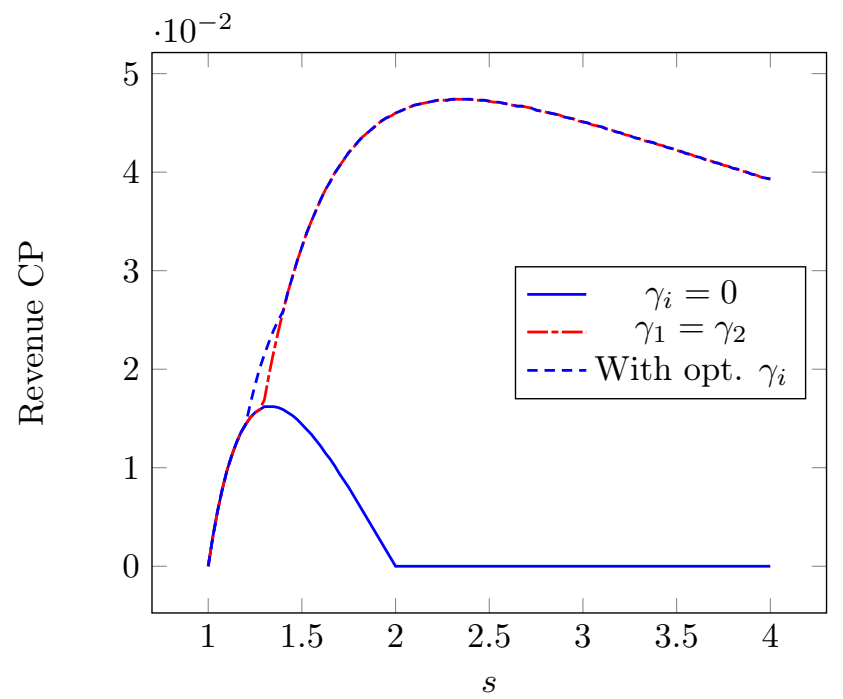

Figure 2: $\mathrm{CP}$ revenue when $\kappa=1.00, \beta=1.00, r_{1}=2.00, r_{2}=1.00, p_{1}=$ $0.16, p_{2}=0.10, q_{1}=0.20, q_{2}=0.10, \alpha=1.00$

there exists an optimal advertising level, which is the same for the equal and differentiated sponsoring cases, around $s=2.35$; it is smaller at $s=1.3$ in case of full neutrality.

ISP revenues are displayed in Figure 3. Remark that the gap between revenues tends to be smaller with sponsoring rather than without sponsoring. Equal sponsoring always gives the best output for both ISPs, something not that intuitive.

Finally, Figure 4 illustrates that user welfare is never worse than with no sponsoring (for this set of parameters), and that the best sponsoring strategy from a regulator point of view depends on the advertising level. Sponsoring also means an associated optimal advertising level, while no advertising is better when there is no sponsoring at all.

\section{Game between ISPs}

As said previously, we assume that subscription prices are fixed (by the market and/or the regulator). As a consequence, ISPs only play with a single parameter, the per-unit-of-volume price $q_{i}$.

Figures 5 to 7 display the best responses of ISPs for the three sponsoring policies. 




Figure 3: ISP revenues when $\kappa=1.00, \beta=1.00, r_{1}=2.00, r_{2}=1.00, p_{1}=$ $0.16, p_{2}=0.10, q_{1}=0.20, q_{2}=0.10, \alpha=1.00$

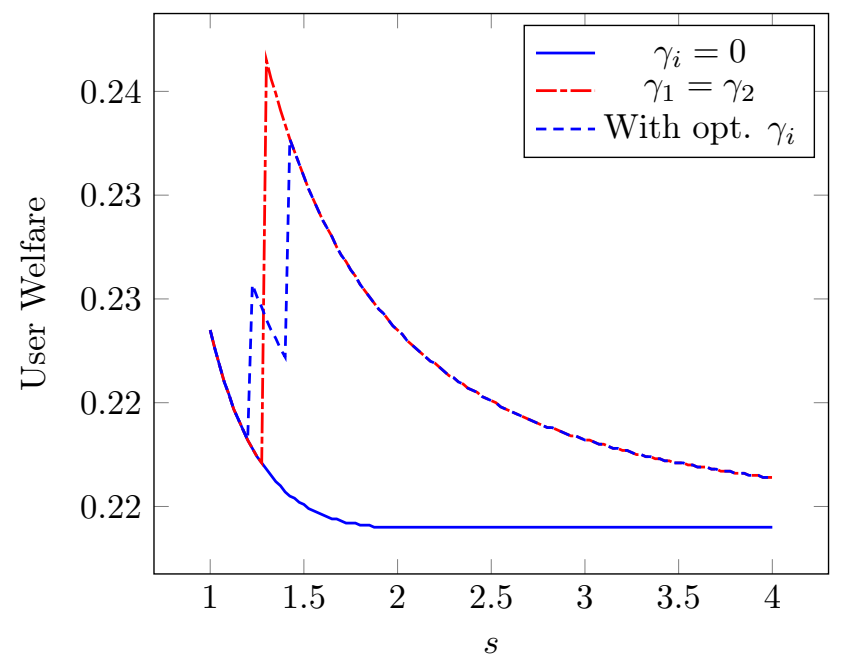

Figure 4: User Welfare when $\kappa=1.00, \beta=1.00, r_{1}=2.00, r_{2}=1.00, p_{1}=$ $0.16, p_{2}=0.10, q_{1}=0.20, q_{2}=0.10, \alpha=1.00$ 


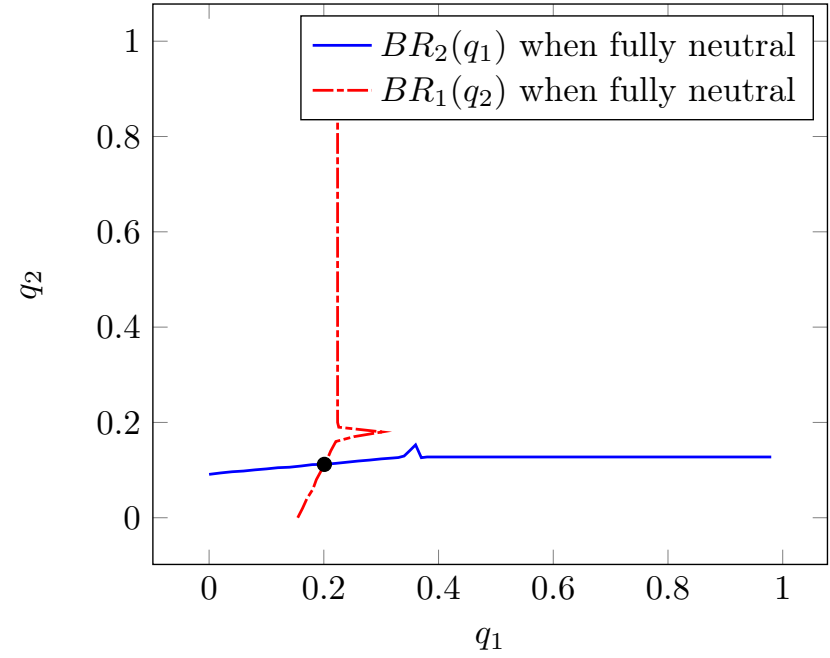

Figure 5: Best responses of ISPs, with $\beta=1.00, r_{1}=2.00, r_{2}=1.00, p_{1}=$ $0.16, p_{2}=0.10, \alpha=1.00$

A Nash equilibrium point (that is, in the non-cooperative game theory context, a situation where no ISP can improve its utility by unilaterally changing its strategy [5]) is a point where best-response curves intersect. We can see such an equilibrium for each sponsoring context.

Figure 5 displays surprising "peaks" on best responses. Looking more closely, it does not seem to be an artifact of the numerical analysis algorithm. Actually, specifically looking at the best response of ISP $1, B R_{1}\left(q_{2}\right)$, when $q_{2}=0.3$ leads to $s=1.14$ and a quite sudden drop to $s=1.089$ for $q_{2}=0.305$ (with respect to what happens before and after those values), leading to the non-monotonous behavior.

The "problem" comes from there being two local maxima in the curves of CP revenue in terms of the advertising level $s$ as illustrated in Figure 8: there is for a short period a change in the local optimum yielding the global optimum, resulting in the discontinuous behavior.

On Figure 7, when differentiated sponsoring is allowed; we only get one Nash equilibrium represented by the point at the limit of the blue segment. It actually corresponds exactly to the one with equal sponsoring. The parts where the curves seem to coincide are actually one curve just below the other, 


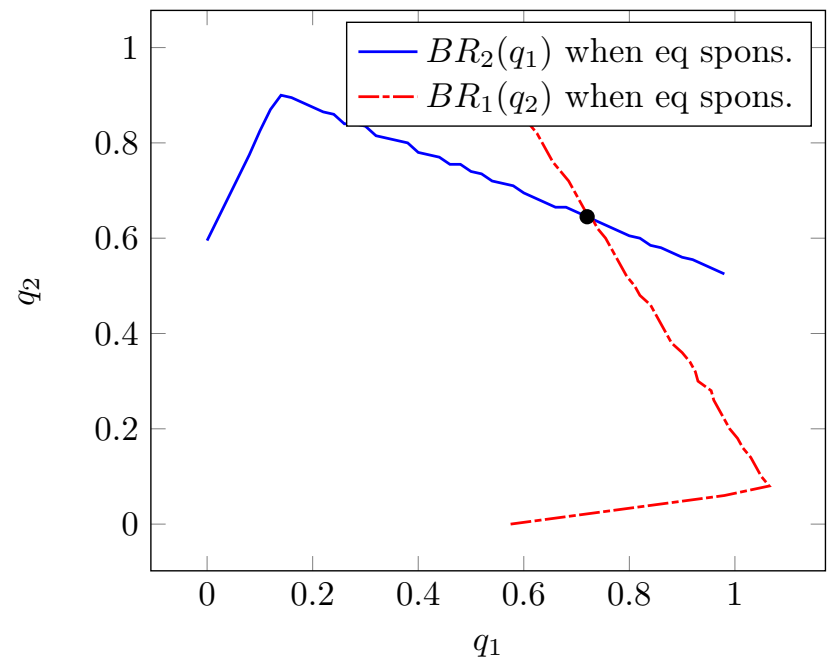

Figure 6: Best responses of ISPs, with $\beta=1.00, r_{1}=2.00, r_{2}=1.00, p_{1}=$ $0.16, p_{2}=0.10, \alpha=1.00$

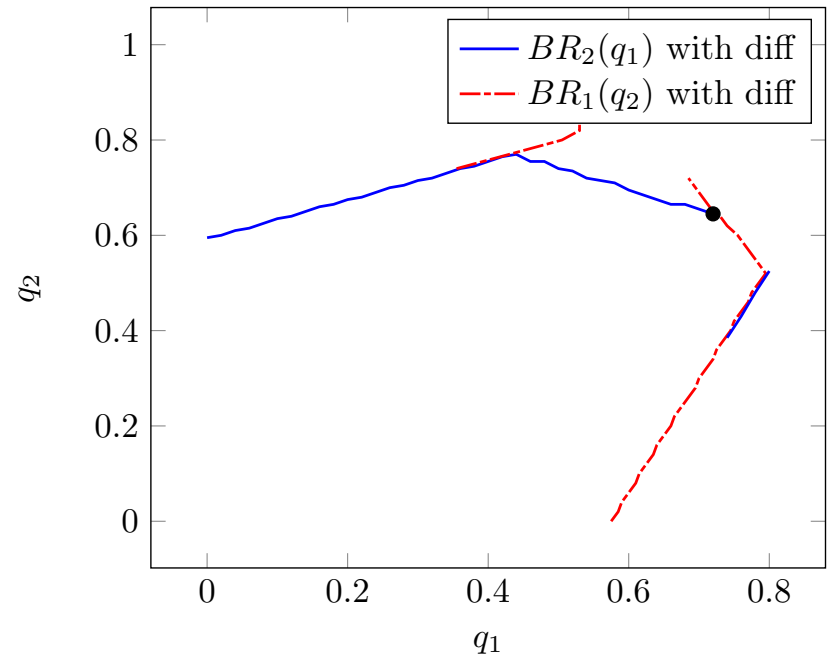

Figure 7: Best responses of ISPs, with $\beta=1.00, r_{1}=2.00, r_{2}=1.00, p_{1}=$ $0.16, p_{2}=0.10, \alpha=1.00$ 


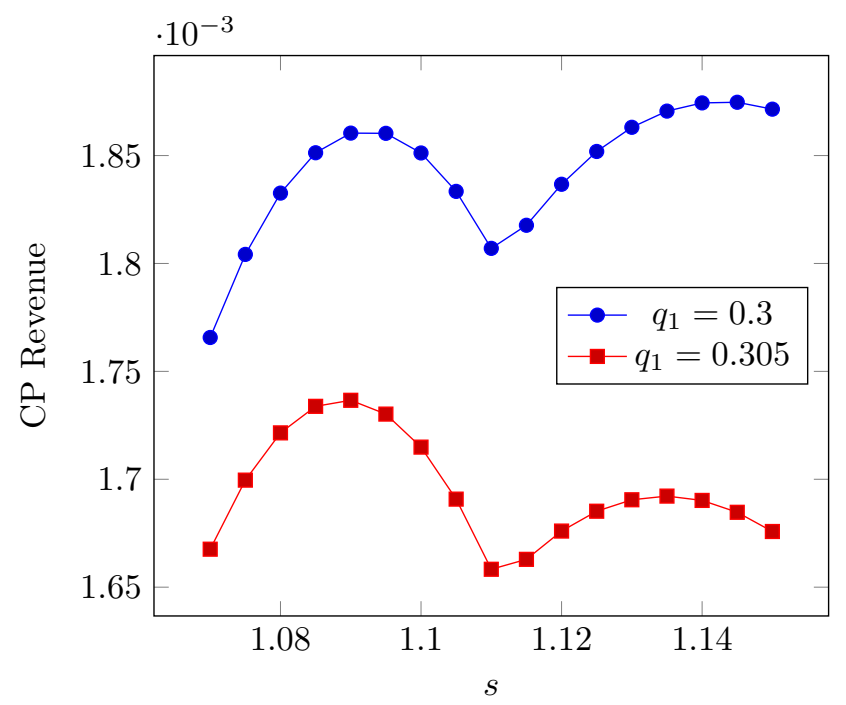

Figure 8: CP revenue in terms of $s$ for two values of $q_{1}$, with $q_{2}=0.18, \beta=$ $1.00, r_{1}=2.00, r_{2}=1.00, p_{1}=0.16, p_{2}=0.10, \alpha=1.00$

corresponding to a price war. In other words, we do not have an equilibrium there, ISPs just reduce their prices a little to attract more revenue, resulting in a slide along the best response curves. Discontinuities correspond to changes of sponsoring strategies. For example on the blue curve corresponding to $B R_{2}\left(q_{1}\right)$, the first segments up to $q_{1}=0.72$ correspond to $\gamma_{1}=\gamma_{2}=1$ while after that we have $\gamma_{1}=0$ and $\gamma_{2}=1$.

Giving explicitly the numerical results, a Nash equilibrium is obtained at:

- With full neutrality, $\left(q_{1}, q_{2}\right)=(0.201,0.112)$

- with equal sponsoring, $\left(q_{1}, q_{2}\right)=(0.720,0.645)$

- with differentiated sponsoring, $\left(q_{1}, q_{2}\right)=(0.720,0.645)$.

We can then compare the output at the optimal prices in Table 4.

\begin{tabular}{c|c|c|c|c|c|c|c} 
Strategy & $\left(q_{1}, q_{2}\right)$ & $\left(\gamma_{1}, \gamma_{2}\right)$ & $s$ & CP & ISP1 & ISP2 & UW \\
\hline Full neut. & $(0.201,0.112)$ & $(0,0)$ & 1.304 & 0.0141 & 0.0534 & 0.0724 & 0.2163 \\
Equal Sp. & $(0.720,0.645)$ & $(1,1)$ & 6.242 & 0.0067 & 0.0615 & 0.084 & 0.215 \\
Diff. Sp. & $(0.720,0.645)$ & $(1,1)$ & 6.242 & 0.0067 & 0.0615 & 0.084 & 0.215
\end{tabular}

Table 1: Output at optimal points 
One can check that outputs are the same for the two sponsoring possibilities because both lead to full sponsoring. The consequence is much more advertisement, but higher volume prices (not experienced by users but paid by the $\mathrm{CP}$ ). The CP is actually not gaining from sponsoring, its revenue being halved; it clearly suffers from not being the leader of the game (that is, not deciding first and anticipatively as opposed to ISPs). ISPs on the other hand benefit from sponsoring due to increased volume prices, even despite price competition. This justifies the motivation of ISPs to develop platforms for sponsoring capabilities. Finally, we can note that sponsoring does not alter much user welfare (only at the third digit), hence a regulator with users satisfaction as primary goal should not necessarily prevent sponsoring, but should monitor it to make sure that it does not reduce satisfaction with another set of parameters.

\section{Conclusions}

This paper addresses a currently important question asked to regulators: should content providers be prevented from sponsoring data and is it a valid question in the network neutrality debate? Regulators worldwide give different answers, some forbidding it, others accepting it, while the remaining ones just want to monitor the activity before making a decision. It illustrates the need to design models to better understand the implications of sponsoring on the various economic actors and on the society.

We have designed in this paper such a model encompassing competition between wireless service providers and advertising. We have illustrated that, contrary to a priori ideas, sponsoring can be beneficial to network providers and not to content providers, while not leading to a significant change for users in terms of satisfaction (gaining in terms of volume data but at the expense of more advertisements). This shows a need for more discussions on the topic.

As future directions of research, we would like to investigate what happens if the content provider is integrated with a network provider (something more and more happening with network providers offering services or content providers trying to get their own network). According to our preliminary results in a competitive context, this seems to lead to no charge for content (so-called zerorating) at the integrated network provider. Another line of research we are interested in is whether sponsored data prevents innovation (that is, creates an entry barrier to newcomers) and competition between content providers.

\section{References}

[1] M. Cho and M. Choi. Pricing for mobile data services considering service evolution and change of user heterogeneity. IEICE Transactions on Communications, E96-B(2):543-552, 2013. 
[2] C. Joe-Wong, S. Ha, and M. Chiang. Sponsoring mobile data: An economic analysis of the impact on users and content providers. In Proc. of INFOCOM, Hong-Kong, China, 2015.

[3] B. Jullien and W. Sand-Zantman. Internet Regulation, Two-Sided Pricing, and Sponsored Data. Tse working papers, Toulouse School of Economics (TSE), 2017.

[4] P. Maillé, P. Reichl, and B. Tuffin. Internet governance and economics of network neutrality. In A. Hadjiantonis and B. Stiller, editors, Telecommunications Economics - Selected Results of the COST Action IS0605 EconTel, pages 108-116. Lecture Notes in Computer Science 7216, Springer Verlag, 2012.

[5] P. Maillé and B. Tuffin. Telecommunication Network Economics: From Theory to Applications. Cambridge University Press, 2014.

[6] P. Maillé and B. Tuffin. Users facing volume-based and flat-rate-based charging schemes at the same time. In 8th Latin American Network Operations and Management Symposium (LANOMS), pages 23-26, Joao Pessoa, Brazil, October 2015.

[7] P. Maillé and B. Tuffin. Analysis of Sponsored Data in the Case of Competing Wireless Service Providers. In NETGCOOP 2018 - 9th International Conference on Network Games, Control and Optimization, pages 1-16, New York City, United States, November 2018.

[8] H. Pang, L. Gao, Q. Ding, and L. Sun. When data sponsoring meets edge caching: A game-theoretic analysis. CoRR, abs/1709.00273, 2017.

[9] R. Somogyi. The economics of zero-rating and net neutrality. CORE Discussion Papers 2016047, Université catholique de Louvain, Center for Operations Research and Econometrics (CORE), 2016.

[10] P. Vyavahare, J. Nair, and D. Manjunath. Sponsored data: On the Effect of ISP Competition on Pricing Dynamics and Content Provider Market Structures. In Submitted to MobiHoc'19, July 2019, Catania Italy, 2019.

[11] Z. Xiong, S. Feng, D. Niyato, P. Wang, and Y. Zhang. Competition and Cooperation Analysis for Data Sponsored Market: A Network Effects Model. ArXiv e-prints, November 2017.

[12] L. Zhang, W. Wu, and D. Wang. Sponsored data plan: A two-class service model in wireless data networks. SIGMETRICS Perform. Eval. Rev., 43(1):85-96, June 2015. 